\title{
The Property of Rationality: A Guide to What Rationality Requires?
}

\author{
Julian Fink ${ }^{1}$
}

\begin{abstract}
Can we employ the property of rationality in establishing what rationality requires? According to a central and formal thesis of John Broome's work on rational requirements, the answer is 'no' - at least if we expect a precise answer. In particular, Broome argues that (i) the property of full rationality (i.e. whether or not you are fully rational) is independent of whether we formulate conditional requirements of rationality as having a wide or a narrow logical scope. That is, (ii) by replacing a wide-scope requirement with a corresponding narrow-scope requirement (or vice versa), we do not alter the situations in which a person is fully rational. As a consequence, (iii) the property of full rationality is unable to guide us in determining whether a rational requirement has a wide or a narrow logical scope. We cannot resolve the wide/narrow scope debate by appealing to a theory of fully rational attitudes. This paper argues that (i), (ii) and (iii) are incorrect. Replacing a wide- with a corresponding narrow-scope requirement (or vice versa) can alter the set of circumstances in which a person is fully rational. The property of full rationality is therefore not independent of whether we formulate conditional requirements of rationality as having a wide or a narrow logical scope. As a consequence, the property of full rationality can guide us in determining what rationality requires - even in cases where we expect a precise answer.
\end{abstract}

\section{Advancing the debate}

The debate on the content and nature of rational requirements that govern attitudinal coherence faces a serious obstacle. ${ }^{2}$ On the one hand, most parties to the debate agree about numerous combinations of attitudes ${ }^{3}$ that violate a requirement of rationality. For example, if you hold a pair of contradictory beliefs or intentions, fail to intend a means you acknowledge as necessary to your ends, have no intention of doing what you believe you ought to do, hold intransitive preferences, or assign inconsistent probabilities to states of affairs, you violate a requirement of rationality.

On the other hand, there is no unified agreement on how we should formulate the requirements that are violated in the above examples. Philosophers disagree about the logical form of rational requirements and, in particular, about whether those requirements are best formulated as requirements with

\footnotetext{
${ }^{1}$ Department of Philosophy, University of Berne, Länggassstrasse 49a, CH-3012 Bern, Switzerland.

2 I assume that rationality is reducible to a particular kind of (structural) coherence among a person's attitudes (cf. for example Scanlon 2007; Broome 2013). I have argued elsewhere that this kind of coherence can be cashed out in terms of the possibility of attitudinal success (Fink 2014 and ms). However, we should also acknowledge a competing (and, it seems, increasingly popular) conception according to which rationality consists in responding correctly to particular normative reasons (see, for example, Lord 2014a and forthcoming; Kiesewetter 2013). For detailed criticism, see Broome 2007d and 2013a. I have criticised versions of this view in Fink (2014 and ms).

${ }^{3}$ Throughout this paper, I use 'attitude' to include the lack of an attitude.
} 
conditional contents or as conditionals whose consequents express a rational requirement.

Consider an illustration of this problem. Suppose you are akratic: you have no intention to $X$ even though you believe that you ought to $X$. I assume that you violate a requirement of rationality. ${ }^{4}$ But which precise requirement do you violate? How should we formulate it?

The answer to this question is not clear. There are at least two plausible formulations, both of which do an equally good job of generating the violation. According to the first formulation, rationality simply prohibits the combination of the two attitudes. What is required is that you not [believe that you ought to $X$ and not intend to $X$ ]. Or, put in its equivalent material conditional form:

(W) Necessarily: rationality requires of you that [(you believe you ought to $X) \rightarrow($ you intend to $X)$ ],

where $\rightarrow$ represents a material conditional. In (W) 'rationality requires' has a wide conditional scope: it governs the entire material conditional '(you believe you ought to $X) \rightarrow$ (you intend to $X$ )'. This guarantees that by believing that you ought to $X$ without intending to $X$, you violate a rational requirement. (The conditional content of the requirement then turns out to be false.) It also guarantees that you can satisfy this requirement by either intending to $X$ or not believing that you ought to $X$. (The content of the requirement then turns out to be true.) Accordingly, (W) represents a first prima facie plausible requirement formulation that would ensure the irrationality of akrasia.

According to the second formulation, 'rationality requires' does not govern a material conditional. Instead, it says that either it is not the case that you believe that you ought to $X$ or rationality requires you to intend to $X$. Or, again, expressed with the help of a material conditional:

(N) Necessarily: (you believe you ought to $X$ ) $\rightarrow$ [rationality requires of you that (you intend to $X$ )].

In (N) 'rationality requires' has a narrow conditional scope: it governs only the consequent of the material conditional '(you believe you ought to $X$ ) $\rightarrow$ (you intend to $X)^{\prime}$ '. This also ensures that akrasia is irrational. Your belief that you ought to $X$ entails that you are rationally required to intend to $X$. But, being akratic, you do not intend to $X$. Thus, you violate a requirement. You can satisfy this requirement in only one way, i.e. by intending to $X$. $(\mathrm{N})$ represents a second prima facie plausible formulation that would ensure the irrationality of $a k r a s i a .^{5}$

\footnotetext{
${ }^{4}$ Within the recent debate, see, for example, Broome (2013a, 2013b), Brunero (2013), and Coates (2013) and Reisner (2013) on the irrationality of akrasia.

${ }^{5}$ Here is a third (but implausible) formulation:
} 
Which formulation should we prefer: $(W)$ or $(N)$ ? Also, which formulation should we employ as a model for stating other conditional requirements of rationality? In the current debate, these are open questions. Good arguments are presented for and against both (types of) formulations. ${ }^{6}$ But are these arguments really worth the effort? Is the question whether we use (W)- or (N)type formulations to represent a rational requirement important?

A close reading of the current debate suggests that it might well be. Indeed, there are good reasons to view the difference between (W)- and (N)-type requirements as in some sense fundamental to the nature of rational requirements. For example, the choice between either (W)- or (N)-type requirements is thought to influence whether the requirements of rationality ..."

(i) ... give us genuinely symmetric options regarding how we can satisfy them (Schroeder 2004); ${ }^{7}$

(ii) ... are prone to issue contradictory requirements (Broome 2007a, 2007b, 2013a; Kolodny 2007b); ${ }^{8}$

(iii) ... pick out necessary conditions for being fully rational (Broome 2007b); ${ }^{9}$

(iv) ... can guide the formation of attitudes (Reisner 2009); ${ }^{10}$

(v) ... are apt for constituting standards for correct reasoning (Kolodny 2005, 2007b; Broome 2013a). ${ }^{11}$

$\left(\mathrm{N}^{*}\right) \quad$ Necessarily: (you do not intend to $\left.X\right) \rightarrow$ [rationality requires of you that (you do not believe that you ought to $X)$ ].

$\left(\mathrm{N}^{*}\right)$ says that not intending to $X$ suffices to put you under a rational requirement not to believe that you ought to $X$. But this is surely implausible. The absence of an intention to $X$ is certainly not (conclusive) evidence of its not being the case that you ought to $X$. I will therefore not include $\left(\mathrm{N}^{*}\right)$ in my discussion.

${ }^{6}$ See in particular Broome (1999, 2004, 2007a, 2007b, and 2013a), Brunero (2010, 2012), Kolodny (2005, 2007a), Rippon (2011), Schroder (2004), Shpall (2013), and Way (2010, 2011).

${ }^{7}$ Suppose you believe you ought to $X$, but you have no intention of $X$-ing. If (W) is correct, there are two genuinely symmetrical options when it comes to satisfying the requirement. You can intend to $X$ or you can be such that you do not believe that you ought to $X$. If $(N)$ is correct, there is only one option, i.e. intending to $X$. Not believing that you ought to $X$ is then not an option when it comes to satisfying the violated requirement. On the question of symmetry, see Schroeder (2009), Kolodny (2005), Brunero (2010, 2012), Bedke (2009), and Way (2010).

${ }^{8}$ Suppose you believe you ought to $X$, and you believe you ought to not- $X$. If $(\mathrm{N})$ is correct, rationality issues a set of contradictory requirements upon you. Rationality then requires you to intend to $X$, and it requires you to intend to not- $X$. (W) has no such implication.

${ }^{9}$ Suppose you hold only one irrational combination of attitudes: you believe you ought to $X$, but you lack an intention to $X$. If $(\mathrm{N})$ is correct, you are rationally required to intend to $X$. However, it is clear that 'You intend to $X$ ' is not strictly a necessary condition for becoming fully rational. There are conceivable situations in which you could also become fully rational by dropping your belief that you ought to $X$. By contrast, the content of (W) seems to state a genuinely necessary condition for full rationality. You can be fully rational only if (you intend to $X$ or do not believe that you ought to $X$ ).

${ }^{10}(\mathrm{~N})$-type requirements can guide the formation of attitudes insofar as they pick out a particular attitude that rationality requires you to have; (W)-type requirements, by contrast, do not tell you which particular attitude you are required to have; they leave you with a set of options.

${ }^{11}$ For example, you can reason (correctly, it seems) from the content of a belief that you ought to $X$ to an intention to $X$. You cannot reason, however, from the content of an absent intention to $X$ to 
(vi) ... are attitude sensitive in their application (Broome 2007b; Lord 2014b). ${ }^{12}$

The most important difference, however, lies elsewhere. Many philosophers suppose that rational requirements are in some way normative (Kolodny 2007a, 230; see Broome 2005; 2013a). Interpreted in a minimal ${ }^{13}$ yet non-trivial way, ${ }^{14}$ this is to say that rational requirements entail normative reasons. ${ }^{15}$ Or, more precisely:

Reasons entailment. Necessarily: if rationality requires you to $X$, there is a normative reason for you to $X$.

Should we endorse this entailment? The difference between (W)- and (N)-type requirements is fundamental to answering this question. Consider a logical consequence of conjoining (N) with Reasons entailment. (N) says that, necessarily, if you believe you ought to $X$, then rationality requires you to intend to $X$. Reasons entailment implies that, necessarily, if rationality requires you to intend to $X$, then there is a normative reason for you to intend to $X$. By virtue of transitive implication, this entails:

Implication. Necessarily: if you believe you ought to $X$, then there is a normative reason for you to intend to $X$.

That is: by adopting a belief that you ought to $X$, you guarantee the existence of a normative reason to intend to $X$. Should we accept Implication?

According to a central finding in the philosophy of reasons and rationality, the answer is clearly 'no'. Implication is subject to the so-called 'bootstrapping objection' (Bratman 1987; Broome 2001; Piller 2013). It entails that for any $X$ (e.g. 'driving drunk', 'becoming a terrorist', etc.) you can create a reason to intend to $X$ simply in virtue of adopting a belief that you ought to $X$. Put succinctly, Implication licences the creation of normative reasons where there aren't any.

suspending the belief that you ought to $X$, because an absent intention to $X$ has no content with which you can reason. $(N)$, unlike (W), seems to capture this fact insofar as once you believe you ought to $X$ (and you do not intend to $X$ ), you can only satisfy (N) by forming an intention to $X$, not by suspending your belief that you ought to $X$.

${ }^{12}$ If $(N)$ is correct, then you can trigger the application of a requirement to intend to $X$ by believing that you ought to $X$. If $(\mathrm{W})$ is correct, the application of the requirement is not in any way sensitive to whether or not you believe that you ought to $X$.

${ }^{13}$ See Broome (2007c, pp. 162-5) on why this is a minimal or 'weak' version of the view that rational requirements are normative.

${ }^{14}$ There is, of course, a trivial sense in which rationality '[...] is automatically normative [...]. Rationality is a system of requirements or rules. It therefore sets up a notion of correctness: following the rules is correct according to the rules. That by itself makes it normative in one sense, because in one sense 'normative' simply means to do with norms, rules or correctness. Any source of requirements is normative in this sense. For example, Catholicism is. Catholicism requires you to abstain from meat on Fridays. This is a rule, and it is incorrect according to Catholicism to eat meat on Fridays. So Catholicism is normative in this sense' (Broome 2007c, p. 162).

${ }^{15}$ See Southwood (2008) and Reisner (2011) for attempts to explain and defend this view. 
Let me illustrate this point. Suppose you have no normative reason to intend to kill yourself. Nothing speaks in favour of such an intention. You also do not believe that you ought to kill yourself. Suppose now that, as a result of someone's spiking your coffee with a dangerous pill, you come to believe that you ought to kill yourself. Does this suffice to create a normative reason to intend to kill yourself? That is, can we create a reason to intend to $X$ by merely adopting the view that we ought to? Evidently, the answer is 'no'. A mere belief that you ought to kill yourself cannot 'bootstrap' such a reason into existence. It cannot create reasons where there are none. In general, reasons for intending to $X$ depend on whether (intending to) $X$ is good or choiceworthy (in the relevant sense). They do not depend on whether one judges $X$ to be good, choiceworthy, or obligatory.

The consequence of this is plain: Implication states an untenable entailment relation between what you believe you ought to do and what reasons there are. Since Implication is a logical consequence of conjoining $(\mathrm{N})$ with Reasons Entailment, at least one of these two propositions must be incorrect too.

Unlike (N), (W) does not give rise to incredible bootstrapping when conjoined with Reasons entailment. Suppose, necessarily, there is a normative reason for you to satisfy (W). This does not imply that your belief that you ought to kill yourself entails a reason to intend to kill yourself. Instead, it implies that there must be a reason to satisfy the following relation: either it is not the case that you believe that you ought to kill yourself or you intend to kill yourself. This is a reason to be ought-belief/intention coherent. It is not a reason to have a particular intention. Hence, accepting (W) and Reasons entailment does not force one to embrace a kind of bootstrapping of normative reasons that goes beyond a normative reason for being coherent.

In sum: conjoining $(\mathrm{N})$ and Reasons entailment entails Implication. By contrast, conjoining (W) and Reasons entailment does not entail Implication. Since Implication represents an unacceptable form of bootstrapping, Reasons entailment does not seem tenable in conjunction with a regime of (N)-type requirements. Thus, in order to advance the debate on the normativity of rational requirements, we first need to settle the choice between (W)- and (N)type requirements. We need to devise a method that tells us whether 'rationality requires' takes a wide or a narrow logical scope in formulating conditional requirements of rationality.

How can we choose between (W) and (N)? In general: how can we identify what rationality requires and when it requires something of us? Not many systematic approaches have been put forward. Broome, and others, find themselves '... forced to appeal largely to our intuitions' (Broome 2013a, p. 150). However, in his recent work (2007a, 2007b, 2013), Broome argues against the very possibility of a seemingly promising strategy. I will call it the 'property strategy'. 
Roughly, the property strategy says that we can employ the property of full rationality in deciding what rationality requires of us and when it does so.

This strategy has three steps: first, we develop a theory about which combinations of attitudes and mental processes are in/consistent with having the property of full rationality. Second, we determine whether (or which) (W)or (N)-type requirements are (more) conducive to our theory of fully rational attitudes. And finally, we choose between (W)- and (N)-type requirements on the basis of which logical form fits best with our theory of fully rational attitudinal combinations and mental processes.

Broome views this strategy as futile. He argues this as a technical point (2007a, pp. $363-4 ; 2013$, p. 134): as far as the property of full rationality is concerned, $(\mathrm{W})$ and $(\mathrm{N})$ turn out to be equivalent. I shall call this 'property equivalence'. That is, the combinations of attitudes under which you are fully rational under a regime of $(\mathrm{N})$-type requirements are identical to the combinations of attitudes under which you are fully rational under a regime of corresponding (W)-type requirements. Thus, replacing an (N)-type requirement with a (W)-type requirement (or vice versa) does not affect the combinations of attitudes that are consistent with full rationality. Any attempt to decide between $(\mathrm{N})$ and $(\mathrm{W})$ by considering the property of full rationality is thus destined to fail.

However, Broome's rejection of the property strategy is too quick. In this paper, I will argue that we should not dispense with the property of rationality as a guide in settling the debate between (W)- and (N)-type requirements. Replacing an (N)-type with a (W)-type requirement can indeed alter the combinations of attitudes that are consistent with having the property of full rationality. This does not, of course, undermine Broome's own defence of the wide-scope form of conditional requirements of rationality. Yet it shows that we should make use of the property of rationality in settling the debate on the scope of rational requirements.

Part of what makes Broome's argument authoritative is that he tries to establish property equivalence with a formal theorem and a corresponding proof (2007a, pp. 369-70; 2007b, pp. 39-40; 2013a, p. 148). Although Broome's theorem is correct (as I show in $\S 6$ ), it fails to establish what he intends to establish, i.e. property equivalence. In particular, the theorem is premised on the implicit but implausible exclusion of a particular entailment relation that may hold between rational requirements. The theorem excludes, for instance, the existence of requirements that prohibit intending that $X$ if one knows that $X$ will lead one to have an attitude one is rationally required not to have. So, while Broome manages to prove property equivalence for a system of rational requirements that excludes such requirements, I will argue that there is no justification for excluding them. Broome is thus unable to sustain his methodological point. There are situations in which we can decide between (W)- and (N)-type requirements by considering attitudinal combinations that are fully rational. Thus the property of rationality might help us to make 
progress on some of the questions that have traditionally been linked to the scope of rational requirements.

This paper proceeds as follows. $\S 2$ introduces the semantic framework for rational requirements that Broome employs in making his equivalence claim. $\S 3$ defines Broome's equivalence claim, i.e. the claim that the property of full rationality remains unaffected by replacing a (W)-type with an (N)-type requirement (or vice versa). $\S 4$ and $\S 5$ advance an argument against property equivalence. I will put forward a counterexample according to which one is fully rational under a (W)-type requirement, but not so under a corresponding (N)type requirement. $\S 6$ turns to Broome's formal theorem, which purports to vindicate property equivalence. I shall demonstrate that Broome's theorem fails to justify property equivalence, with the upshot that the property of rationality is still available to us as a possible guide in constructing the logical form of rational requirements. There are situations in which the property strategy can determine whether (N)- or (W)-type requirements represent rational requirements correctly, thereby helping us, for instance, to form a correct view on whether the bootstrapping objection poses a threat to the normativity of rational requirements.

\section{The code of rationality}

How can we decide whether (W)- or (N)-type formulations correctly represent a conditional requirement of rationality? Two things need to be taken into account: (i) 'rationality requires' is by and large '... a philosopher's phrase of art' (Kolodny 2005, p. 515); and (ii) (W)- and (N)-type requirements seem to differ significantly with regard to their logical properties. Consequently, as a first step towards deciding on the correctness of (W)- and (N)-type requirements, we need to work towards a semantics of 'rationality requires' (Broome 2007a, p. 361).

Broome proposes a general semantic framework for rational requirements (2007a). His framework is in fact so general that it could be adopted for other systems of requirements, such as morality or prudence (Broome 2007b and 2013a). Nonetheless, Broome employs this framework to render precise key notions concerning rationality and its requirements and to expose the exact difference between (W)- and (N)-type requirements. He also uses the semantic framework in his proof that (W)- and (N)-type requirements are equivalent with respect to the property of rationality.

In essence, Broome's framework rests on two general stipulations (2007a, pp. 361-3). First, for every possible world $w$, there is a set of propositions that rationality requires of you. That is, at $w, p$ is a member of the set required propositions if and only if, at $w$, rationality requires of you that $p$. Second, there is a function that relates worlds to sets of required propositions. 
This function is called the 'code of rationality' (or just 'code' hereafter). A code is a mapping from worlds to sets of required propositions. A complete code thus determines for all worlds $w$ and all propositions $p$ whether or not $p$ is a member of the set of required propositions at $w$. Less technically, a code specifies what rationality requires of you and when it does so.

Broome's general code semantics also allows us to define when a person possesses the property of full rationality. That is, at $w$, you are fully rational if and only if, at $w$, every proposition in $w$ 's set of required propositions - call it $R P(w)$ - is true at $w$ (Broome 2007a, p. 362).

In assigning required propositions to worlds, a code will satisfy a variety of constraints. Some constraints will be purely formal. Since a code is a function, it cannot assign two different sets of required propositions to the same world. Other constraints will stem from the logic of rational requirements. A correct code may, for example, satisfy a 'no-conflict' constraint, as it exists in standard deontic logic (Broome 2007a, p. 365; 2013a, p. 122). That is, necessarily, if, at $w$, $p$ is an element of $R P(w)$, then it is not the case that not- $p$ is an element of $R P(w)$. Other constraints will be more substantive. I assume, for instance, that if, at $w$, you possess no capacity for rationality (i.e. logical and reasoning abilities), then $R P(w)$ will be the empty set. ${ }^{16}$

Some constraints will represent individual requirements. I will thus call them 'individual constraints'. Put roughly, an individual constraint signifies how a code injects a particular proposition into $R P(w)$. Individual constraints allow us to express precisely when a code instantiates a wide- and/or a narrow-scope requirement. In principle, a code instantiates a narrow-scope requirement if and only if (NC) describes how a code injects a proposition into the set of required propositions. This description reads as follows:

(NC) For all $w:(p \in w) \rightarrow[q \in R P(w)]$,

where $w$ represents a possible world, $p$ and $q$ stand for individual propositional attitudes of yours, $\in$ reads as 'is an element of', and $R P(w)$ represents the set of rationally required propositions at $w$. (NC) says that for all possible worlds in which $p$ holds true, it also holds true that $q$ is a proposition that is required by rationality.

Analogously, a code instantiates a wide-scope requirement if and only if (WC) describes how a code injects a proposition into the set of required propositions. This description reads as follows:

(WC) For all $w:(p \rightarrow q) \in R P(w)$.

\footnotetext{
${ }^{16}$ I elaborate this point in Fink (2014).
} 
(WC) says that it holds for all possible worlds that the truth of the material conditional $p \rightarrow q$ is rationally required of you.

In sum, the code semantics helps us to expose the precise difference between wide- and narrow-scope requirements. (WC)-type requirements require the truth of the material conditional $p \rightarrow q$ (i.e. either ( $p$ and $q$ ), or ( $p$ and not- $q$ ), or (not- $p$ and not- $q$ )). They do so at all possible worlds. (NC)-type requirements require the truth of $q$. They do so at all worlds in which $p$ holds true.

\section{Property equivalence}

I now turn to the claim that (WC)- and (NC)-type requirements are equivalent when it comes to the property of full rationality. Consider two individual propositional attitudes, $p$ and $q$. Suppose that the conjunction of [ $p$ and not- $q$ ] (but neither $p$ nor not- $q$ individually) necessarily results in your violating a rational requirement. (As indicated in $\S 1$, you could, for example, think of $p$ as standing for 'You believe that you ought to $X$ ' and $q$ as standing for 'You intend that you $X^{\prime}$.) Which individual constraint should we assign to a code in order to guarantee this?

As far as this point is concerned, we have a choice: we could assign either (WC) or (NC). Any code that satisfies at least one of these constraints will ensure that whenever [ $p$ and not- $q]$ is an element of $w$, the set of required propositions at $w-R P(w)-$ will contain at least one false proposition. But how can we decide between (WC) and (NC)? That is, which constraint - (WC) or (NC) - correctly represents a conditional requirement?

In answering this question, Broome rejects the strategy of choosing between (WC) and (NC) by considering when one is fully rational. In Rationality Through Reasoning, he emphasises this point as follows:

One putative approach to answering the question about scope will definitely not work. It will do no good to think about the property that corresponds to the source of requirements we are investigating. [...] You might think we could start by working out implications wide-scope and narrow-scope requirements have for the property of [full] rationality. It might turn out that one gives a better account of the property than the other. But actually this is not so. (Broome 2013a, pp. 133-4)

That is to say: we cannot first define the attitudinal combinations that are in/consistent with having the property of full rationality and then choose between (WC) or (NC) on the basis of which formulation best matches our account of fully rational attitudes. This strategy is untenable, Broome argues, because (WC) and (NC) are too similar. (WC) and (NC) are 'property equivalent' when it comes to the property of full rationality:

There is less difference between [(WC) and (NC)] than one might think. Perhaps the most important question a system of rational requirements needs to settle is whether you are [fully] rational-have the property of [full] rationality. It turns out 
that the answer to this question is unaffected by the choice between narrow and wide scope. The proposition that you are rational is the same whichever way a conditional requirement is formulated. Either way, you are rational at exactly the same worlds. (Broome 2007a, p. 363; emphasis added)

In sum, the logical form of a conditional requirement is immaterial to the question of when a person is fully rational. By manipulating a code only to the effect that it satisfies (NC) instead of (WC) (or vice versa), one does not change the set of combinations of attitudes that are consistent with full rationality.

For the sake of precision, let me make this point more formally. Take a code $R_{1}$ - that satisfies (WC) for a pair of propositions $p$ and $q$. That is to say: $R_{1}$ distributes $(p \rightarrow q)$ to the set of required propositions at all worlds. Perform the following operation on $R_{1}$. First, only remove (WC) from $R_{1}$. Thus $R_{1}$ no longer distributes $(p \rightarrow q)$ to the set of required propositions at all worlds. Second, apply (NC) to $R_{1}$. Call the resulting code $R_{2}$. So, $R_{2}$ distributes $q$ to the set of required propositions at all worlds where $p$ holds true. Then $R_{1}$ and $R_{2}$ are 'property equivalent' in the following sense:

Property equivalence. Necessarily: at $w$, you are fully rational under $R_{1}$ if and only if, at $w$, you are fully rational under $R_{2}$.

\section{A counterexample}

As noted above, Property equivalence has not received much critical attention. ${ }^{17}$ This is surprising because its correctness could reduce the significance of the ongoing wide/narrow scope debate. ${ }^{18}$ To the degree that '... the most important question a system of rational requirements needs to settle is whether you are [fully] rational - have the property of [full] rationality' (Broome 2007a, p. 363), Property equivalence threatens the importance of the scope distinction. ${ }^{19}$

However, this lack of critical attention is also a mistake, since Property equivalence is incorrect. By changing a code merely such that it now satisfies (WC) rather than (NC), one can alter the combinations of attitudes that are consistent with being fully rational. So, even when we are only concerned with the property of rationality, the question of scope is highly significant after all.

\footnotetext{
${ }^{17}$ Kolodny (2007b) and Žarnić (2010) represent two exceptions.

${ }^{18}$ Compare, for example, Broome (1999, 2007a, 2013a), Brunero (2010, 2012), Evers (2011), Kolodny (2007b), Rippon (2011), Shpall (2013), and Way (2010).

${ }^{19}$ Niko Kolodny emphasises this point very eloquently:

For years now, it has seemed to Broome and to the rest of us, who have been so stimulated by his work, that there is a crucial difference between the wide and narrow scope. Time and again, Broome has urged us to appreciate this important difference, and by and large we have been convinced. On closer inspection, however, the difference seems almost negligible. (Kolodny 2007b, p. 375)
} 
Consider first an entirely schematic counterexample to Property equivalence. ${ }^{20}$ Compare two codes: $R_{\mathrm{W}}$ and $R_{\mathrm{N}}$. Both satisfy two individual constraints: $R_{\mathrm{W}}$ satisfies (WC) and (LR). (LR) reads as follows:

$$
\text { (LR) For all } w:[q \in R P(w)] \rightarrow[r \in R P(w)] .^{21}
$$

(LR) constrains a code as follows: suppose that, at $w, q$ is among the rationally required propositions. Then, at $w, r$ is also among the rationally required propositions. That is, you cannot be rationally required to $q$ without being required to $r$.

Correspondingly, $R_{\mathrm{N}}$ satisfies (NC) and (LR). $R_{\mathrm{N}}$ is thus the result of only one operation performed on $R_{\mathrm{W}}$, namely the replacement of (WC) with (NC). Accordingly, $R_{\mathrm{W}}$ and $R_{\mathrm{N}}$ fall within the range of Property equivalence. Schematically, the situation is as follows:

\section{Code $\left(\mathbf{R}_{\mathrm{w}}\right)$}

(WC) For all $w:(p \rightarrow q) \in R P(w)$.

$(\mathrm{LR})$ For all $w:[q \in R P(w)] \rightarrow$

$[r \in R P(w)]$.

\section{Code $\left(\mathbf{R}_{\mathbf{N}}\right)$}

(NC) For all $w:(p \in w) \rightarrow[q \in R P(w)]$.

$(\mathrm{LR})$ For all $w:[q \in R P(w)] \rightarrow$

$[r \in R P(w)]$.

Compare $R_{\mathrm{W}}$ with $R_{\mathrm{N}}$. According to Property equivalence, the following proposition should hold true: necessarily, at $w$, you are fully rational under $R_{W}$ if and only if, at $w$, you are fully rational under $R_{N}$. But this is not the case. There is a possible situation in which you are fully rational under $R_{\mathrm{w}}$ and are less than fully rational under $R_{\mathrm{N}}$.

Suppose that, at $w^{\prime},[p, q$, and not-r] signifies a conjunction of your attitudes. Under $R_{\mathrm{N}}$, you cannot be fully rational at $w^{\prime}$. To show this, conjoin (NC) with $p$. This entails that, at $w^{\prime}, q$ is a required proposition. Next, conjoin the fact that $q$ is a required proposition with (LR). This entails that, at $w^{\prime}, r$ is a required

\footnotetext{
${ }^{20}$ Kolodny (2007b, p. 376) also presents an argument against Property equivalence. He argues that Property equivalence fails to hold for 'process requirements', as he puts it. However, Kolodny's counterexample proves incorrect. This is shown in the appendix to this paper.

${ }^{21}$ I have encountered the claim that (LR) is in tension with Broome's code semantics. This is because (LR) fails to specify a unique code. True, a code could satisfy (LR) in various ways. It could satisfy (LR), for example, by virtue of its being necessarily not the case that $q$ is a required proposition at $w$. Alternatively, a code could satisfy (LR) in virtue of the fact that $r$ is a required proposition necessarily. This claim puzzles me, however. Broome's (and my) aim is to establish whether exchanging wide- for narrow-scope requirements (or vice versa) can influence the property of full rationality. To do so, we are in fact forced to formulate constraints on codes that fail to pick out a unique code. For even by making a code behave in accordance with a narrow-scope requirement (i.e. constraining a code so that it satisfies (NC) [i.e. For all $w:(p \in w) \rightarrow[q \in R P(w)])$, one does not specify a unique code. As with (LR), a code can satisfy (NC) in different ways, e.g. by ensuring that $p$ is necessarily not an element of $w$ or by $q$ 's being necessarily required.
} 
proposition. However, at $w^{\prime}$, not-r holds true. So, you cannot be fully rational at $w^{\prime}$ under $R_{\mathrm{N}}$.

This is not so under $R_{\mathrm{W}}$, however. You can be fully rational under $R_{\mathrm{W}}$ at $w^{\prime}$. First, (WC) assigns $p \rightarrow q$ as a required proposition to $w^{\prime}$. The fact that, at $w^{\prime}$, both $p$ and $q$ hold true ensures the truth of $p \rightarrow q$. (LR) says that $r$ is a required proposition at $w^{\prime}$ whenever $q$ is a required proposition at $w^{\prime}$. However, given what we know of $R_{\mathrm{W}}$ and $w^{\prime}$, under $R_{\mathrm{W}}$, there is no need to assume that $q$ is a required propositions at $w^{\prime}$. Also, there is no need to assume that, under $R_{W}, r$ is a required proposition at $w^{\prime}$. So, under $R_{W}$, you can be fully rational at $w^{\prime}$.

Of course, this result requires two things. First, conjoining $p$ and $(\mathrm{WC}$ ) does not entail that $q$ is a required proposition via 'factual detachment'. Second, it is not the case that $p$ is a necessary attitude of yours. I take both conditions to be unproblematic.

Put schematically, 'factual detachment' licenses an inference from

$$
\begin{aligned}
& \text { (i) at } w, ' p \rightarrow q \text { ' is a required proposition } \\
& \text { and (ii) at } w, p \\
& \text { to } \quad \text { (iii) at } w, q \text { is a required proposition. }
\end{aligned}
$$

If this were correct, $[p, q$, and not- $r]$ would not be consistent with being fully rational under $R_{W}$. At $w^{\prime}$, by conjoining $p$ and (WC), $q$ would turn out to be a required proposition. (LR) would then imply that, at $w^{\prime}, r$ is a required proposition. Ad hypothesis, $r$ is not the case at $w^{\prime}$. You would not be fully rational under $R_{\mathrm{w}}$.

Likewise, we need to assume that $p$ is not necessarily true. Otherwise, $q$ (and therefore $r$ ) would again turn out to be required under $R_{W}$ - this time via not 'factual' but rather 'necessary' detachment (Broome 2013a, p. 123), i.e. a plausible inference to (iii) from (i) and

$$
\text { (ii') at } w \text {, necessarily } p \text {. }
$$

As above, $[p, q$, and not-r] would not be consistent with being fully rational under $R_{\mathrm{w}}$.

It is easy to avoid 'necessary detachment'. We are, of course, entitled to stipulate that $p$ stands for a non-necessary attitude. That is, $p$ represents an attitude of yours that you have at some but not all possible worlds. I assume this holds true for most, if not all, attitudes. 
Likewise, 'factual detachment' does not pose a problem either. I assume that it is invalid. First, it resembles an invalid modal inference:

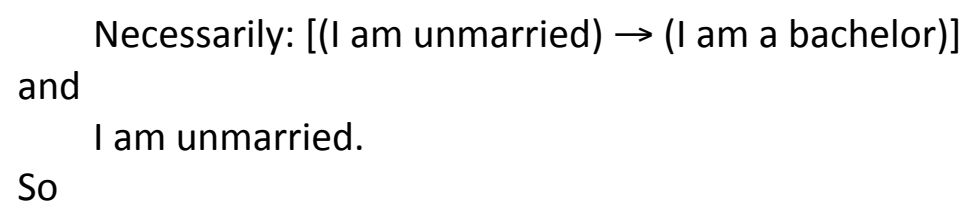

Necessarily: I am a bachelor.

No doubt, this is incorrect (cf. Rippon 2011, pp. 4-5). 'Factual detachment' is also philosophically unattractive. If we were to allow it, (WC)-codes would be as open to the bootstrapping objection as (NC) ones. I therefore reject 'factual detachment'.

The upshot, then, is this: $p, q$, and not-r are jointly consistent with being fully rational at $w^{\prime}$ under $R_{\mathrm{W}}$. However, this is not so under $R_{\mathrm{N}}$. There is a combination of attitudes that permits full rationality under $R_{\mathrm{W}}$ but does not do so under $R_{\mathrm{N}}$. $R_{\mathrm{W}}$ and $R_{\mathrm{N}}$ are not equivalent with respect to the property of full rationality. Property equivalence is incorrect.

\section{Beyond the conceptual result}

So far, I have shown that, conceptually, the choice between wide- and narrowscope requirements is not negligible when it comes to the property of full rationality. Replacing a wide- with a narrow-scope requirement can logically alter the circumstances in which you are fully rational.

This is, I believe, an interesting theoretical result. However, it does not yet imply that there is a correct code for which replacing a wide- with a narrowscope requirement will actually change the circumstances in which you are fully rational under that code. This depends on whether we can find a code for which either (WC) or (NC), taken together with (LR), represents a correct individual constraint. Only then can we guarantee that the property of full rationality can actually help us to determine whether (WC) or (NC) represents a rational requirement correctly.

Recall (LR). It says that one required proposition entails another required proposition. However, you might think that required propositions are not related in this way. Hence, a code that satisfies (LR) cannot represent a correct code of rationality.

This reaction would clearly be ad hoc. It is not unnatural to constrain a code such that a required proposition implies another required proposition. Take a code that injects a conjunction of beliefs - 'You believe that $a$ and you believe that $b^{\prime}-$ into the set of required propositions at all worlds. It seems natural to think that this code will also inject each conjunct - 'You believe that $a$ ' and 'You 
believe that $b^{\prime}$ - into the code of required propositions at all worlds. A required proposition may thus entail another required proposition.

Next, consider a concrete example, where replacing a wide- with a narrowscope requirement changes the circumstances in which you are fully rational. Suppose, at world $w^{*}$, (i) you believe that you ought give up smoking and (ii) you do not believe that it is not the case that you ought to give up smoking. However, (iii) you believe that if you have a conversation with $\operatorname{Simon}^{22}$ (a passionate smoker and a master of persuasion), you will (instantly) believe that it is not the case that you ought to give up smoking. Nevertheless, (iv) you intend to have a conversation with Simon.

Let us construe a code $-R_{\mathrm{W}^{*}}-$ that permits your being fully rational at $w^{*}$. $R_{\mathrm{W}^{*}}$ may, for instance, satisfy the following (WC)-type constraint:

Wide ought-belief consistency. For all $w:[B(O) \rightarrow \neg B(\neg O)] \in R P(w)$,

where $B$ stands for 'You believe that', $O$ for 'You ought to give up smoking', and $\neg$ for negation. Expressed informally, Wide ought-belief consistency says that rationality requires you not to have contradictory ought-beliefs. More formally, it says that, at all possible worlds, the material conditional 'you believe you ought to give up smoking $\rightarrow$ it is not the case that you believe that it is not the case that you ought to give up smoking)' is a rationally required proposition.

Wide ought-belief consistency may constrain $R_{\mathrm{W}^{*}} R_{\mathrm{W}^{*}}$ is a code under which you can be fully rational at $w^{*}$. At $w^{*}$, (i) you believe you ought to give up smoking, and (ii) you have no belief that it is not the case that you ought to give up smoking. So, at $w^{*}$, you satisfy the requirement that wide ought-belief consistency represents.

Consider another possible constraint on $R_{\mathrm{W}^{*}}$ :

Safety. For all $w:\{[\neg B(\neg O) \in R P(w)] \& B[X \rightarrow B(\neg O)]\} \rightarrow[\neg I(X) \in R P(w)]$,

where $I$ stands for 'You intend that', and $X$ stands for 'You have a conversation with Simon'. The general idea behind Safety is that rationality requires you not to intend anything that you believe will bring about a situation that violates a requirement of rationality. That is, suppose 'You do not believe that it is not the case that you ought to give up smoking' is a rationally required proposition. Suppose also that you believe that if you have a conversation with Simon, then you will believe that it is not the case that you ought to give up smoking. Then,

\footnotetext{
${ }^{22}$ In other words: not having a conversation with Simon is a necessary condition for not coming to believe that it is not the case that you ought to give up smoking.
} 
as Safety expresses, 'You do not intend to have a conversation with Simon' is also a rationally required proposition. ${ }^{23}$

As with Wide ought-belief consistency, Safety may constrain $R_{\mathrm{W}^{*}}$. It permits you to be fully rational at $w^{*}$. This requires, of course, that (ii) 'You do not believe that it is not the case that you ought to give up smoking' is not a required proposition at $w^{*}$. For if (ii) were a required proposition at $w^{*}$, Safety would, in conjunction with (iii) and (iv), entail that $R P\left(w^{*}\right)$ contains at least one false proposition at $w^{*}$.

However, we need not assume that (ii) is a member of $R P\left(w^{*}\right)$. First, as I deny factual detachment, conjoining (i) with Wide ought-belief consistency does not imply that (ii) is required at $w^{*}$. Second, I assume that, at $w^{*}$, it is not the case that (i) is a necessary attitude of yours. This prevents (ii)'s being injected into $R P\left(w^{*}\right)$ via necessary detachment. ${ }^{24}$ Safety can hence constrain $R\left(w^{*}\right)$ too.

Moreover, I think that Safety is a plausible constraint on a code of rationality. Consider an analogy with moral requirements. Suppose that racist beliefs are gravely immoral (cf. Appiah 1990; Lengbeyer 2004). Racist beliefs are not only false but also dispositions to act immorally. Thus, if $r$ expresses racist content, then morality requires you not to believe $r$. Suppose now that you believe that having a conversation with William - a persuasive racist - will lead you to form a cluster of racist beliefs. It seems very plausible that in this situation morality requires you not to intend to have a conversation with William. Put generally, morality requires you, among other things, not to intend to engage in behaviour that will make you gravely immoral.

A similar argument suggests itself for Safety and rationality. Rationality, I have already assumed, aims at attitudinal coherence. Roughly, this is to say (as I have argued in detail in Author's paper) that rationality aims at ensuring that your attitudes can simultaneously fulfil their 'constitutive aims' (or 'success conditions'). For example, I assume that beliefs aim constitutively at truth, intentions at implementation, and ought-beliefs at both truth and implementation.

Suppose now that rationality requires you to abstain from believing that it is not the case that you ought to give up smoking. Given my account of coherence, this is the case only if abstaining from having this belief is pivotal to preserving your attitudes' ability simultaneously to fulfil their constitutive aims. Suppose

\footnotetext{
${ }^{23}$ For the sake of simplicity, I have kept a temporal restriction on Safety implicit. Suppose rationality requires you not to have a particular ought-belief - call it $B_{0}$ - between December $1^{\text {st }}$ and December $24^{\text {th }}$. Suppose too that before December $1^{\text {st }}$ and after December $24^{\text {th }}$, you are not required not to have $B_{0}$. Then Safety implies a requirement not to intend $X$ if and only if you believe that $X$ will make you adopt $B_{0}$ between December $1^{\text {st }}$ and December $24^{\text {th }}$ ]. Suppose you believe instead that [ $X$ will make you adopt $B_{0}$ only before December $1^{\text {st }}$ and/or after December $24^{\text {th }}$ ]. Then, I assume, Safety does not imply a rational requirement not to intend to $X$.

${ }^{24}$ Compare $\S 4$ for a brief discussion of 'factual' and 'necessary detachment'.
} 
you believe, however, that having a conversation with Simon will cause you to believe that it is not the case that you ought to give up smoking. Intending to have a conversation with Simon therefore jeopardizes your attitudes' ability to fulfil their constitutive aims. Rationality thus requires you not to have this intention. In general, as in the moral example, rationality requires you not to intend anything you believe will cause you to have an attitude for which it happens to be true that rationally requires you not to have it.

Here is another brief way to consolidate this point. Having a pair of contradictory intentions is one clear way to be incoherent. You cannot implement both intentions. Thus, rationality requires you not to have a pair of contradictory intentions. However, intending to have a pair of contradictory intentions is also a distinctive way of being incoherent. It is to aim to implement something that cannot itself be implemented. This is why, I assume, rationality requires you not to intend to have a pair of contradictory intentions. This lends support to viewing Safety as a plausible requirement of rationality.

I now return to Property equivalence. Recall that $R_{\mathrm{W}^{*}}$ is a code under which you are fully rational at $w^{*}$. Apply the instructions from Property equivalence: remove Wide ought-belief consistency from $R_{\mathrm{W}^{*}}$ and inject its narrow-scope equivalent (i.e. 'narrow ought-belief consistency') into $R_{\mathrm{W}^{*}}$. Call the resulting code $R_{\mathrm{N}^{*}}$. 'Narrow ought-belief consistency' reads as follows:

Narrow ought-belief consistency. For all $w:[B(O) \in w] \rightarrow[\neg B(\neg O) \in R P(w)]$.

Narrow ought-belief consistency says that whenever you believe you ought to give up smoking, 'You do not believe that it is not the case that you ought to give up smoking' is a rationally required proposition. In general, as long as you believe something, rationality requires you not to believe its negation.

In sum, $R_{\mathrm{W} *}$ and $R_{\mathrm{N}^{*}}$ compare as follows:

\section{Code $\left(R_{W^{*}}\right)$}

(Wide ought-belief consistency)

For all $w:[B(O) \rightarrow \neg B(\neg O)] \in R P(w)$.

(Safety)

For all $w:\{[\neg B(\neg O) \in R P(w)] \&$

$B[X \rightarrow B(\neg O)]\} \rightarrow[\neg /(X) \in R P(w)]$.

\section{Code $\left(R_{N^{*}}\right)$}

(Narrow ought-belief consistency) For all $w:[B(O) \in w] \rightarrow[\neg B(\neg O) \in R P(w)]$.

(Safety)

For all $w:\{[\neg B(\neg O) \in R P(w)] \&$ $B[X \rightarrow B(\neg O)]\} \rightarrow[\neg I(X) \in R P(w)]$.

Property equivalence predicts that you are fully rational under $R_{\mathrm{W}^{*}}$ if and only if you are fully rational under $R_{N^{*}}$. However, $w^{*}$ shows this to be incorrect.

I have already shown that you are fully rational under $R_{\mathrm{W}^{*}}$. However, this is not so under $R_{N^{*}}$. Recall $w^{*}$ : (i) you believe that you ought to give up smoking, and 
(ii) you do not believe that it is not the case that you ought to give up smoking. Furthermore, (iii) you believe that if you have a conversation with Simon, you will believe that it is not the case that you ought to give up smoking. Also, (iv) you intend to have a conversation with Simon. Conjoining (i) with Narrow ought-belief consistency of $R_{N^{*}}$ implies that 'It is not the case that you believe that it is not the case that you ought to give up smoking' is a required proposition at $w^{*}$. Conjoining this with the fact that (iii) you believe that [if you have a conversation with Simon, then you believe that it is not the case that you ought to give up smoking], implies, via Safety, that, at $w^{*}$ 'It is not the case that you intend to have a conversation with Simon' is a required proposition. However, ad hypothesis, at $w^{*}$, (iv) you intend to have a conversation with Simon. So while, at $w^{*}$, you are fully rational under $R_{\mathrm{W}^{*}}$, this is not so under $R_{\mathrm{N}^{*}}$.

Again, Property equivalence proves incorrect. ${ }^{25}$ This time, however, it does so for what I take to be a plausible set of codes. This shows that there are situations in which the property of full rationality can actually help us to determine the scope of a conditional requirement of rationality. Wide- and narrow-scope requirements differ practically in more respects than Broome suggests.

\section{Broome's theorem}

This result is surprising. Broome does not defend Property equivalence in passing. Rather, he attempts to prove Property equivalence by formulating a general theorem and a corresponding proof.

This final section turns to Broome's theorem. I argue that it fails to vindicate Property equivalence. Though formally correct, the theorem neither represents nor entails Property equivalence. In fact, the theorem is too weak to support the following two main points: (i) you can replace a wide- with a narrow-scope requirement (and vice versa) without changing the conditions under which you are fully rational; and (ii) '[...] we cannot decide between the wide-scope and narrow-scope formulations by considering when you have the property of rationality' (Broome 2007a, p. 364).

\footnotetext{
${ }^{25}$ Kolodny (2007b, p. 375 , n. 6) claims that 'Broome might have proved a more general claim' than Property equivalence. This is also incorrect, however. Here is Kolodny's claim:
}

Take two codes of rationality according to which (however different they may otherwise be) the proposition that you are rational is the same. Add a narrow-scope conditional requirement to one code and the corresponding wide-scope requirement to the other. Then the proposition that you are rational remains the same. (Kolodny 2007b, p. 375, n. 6)

I shall call this 'addition equivalence'. Both of my counterexamples show that addition equivalence is incorrect. Let $R_{\mathrm{N}}$ and $R_{\mathrm{W}}$ be two codes and assume that: (i) both pick out the same circumstances under which you are fully rational, and (ii) both contain Safety. Add Wide ought-belief consistency to $R_{\mathrm{W}}$ and Narrow ought-belief consistency to $R_{\mathrm{N}}$. As I have demonstrated above, there is a combination of attitudes under which you are fully rational under $R_{\mathrm{W}}$ and not so under $R_{\mathrm{N}}$. 
To see this, first consider Broome's theorem ${ }^{26}$ :

Theorem. Let $R_{1}$ and $R_{2}$ be two codes that are the same except that, for one pair of propositions $p$ and $q, q \in R_{1}(w)$ for all $w$ at which $p$ is true (and this may not be so for $R_{2}$ ) whereas $(p \rightarrow q) \in R_{2}(w)$ for all $w$ (and this may not be so for $R_{1}$ ). Then 'You are [fully] rational' is true under $R_{1}$ at exactly those worlds where it is true under $R_{2}$. (Broome 2007a, p. 369)

To avoid confusion, note that $R_{1}$ and $R_{2}$ represent entire codes. $R_{1}(w)$ and $R_{2}(w)$ stand for corresponding sets of rationally required propositions. Theorem claims the following. Suppose two codes, $R_{1}$ and $R_{2}$, differ only in the following way: for a pair of propositions $p$ and $q, R_{1}$ satisfies (NC) and does not satisfy (WC). Analogously, $R_{2}$ satisfies (WC) and does not satisfy (NC). In all other respects, $R_{1}$ and $R_{2}$ are identical. Then the two codes pick out the same set of worlds at which you are fully rational.

Unlike Property equivalence, Theorem is correct. ${ }^{27}$ It is relatively easy to show this. Let $R_{1}$ and $R_{2}$ differ in terms of precisely the two properties that Theorem assigns to $R_{1}$ and $R_{2}$. That is: $R_{1}$ satisfies (WC) and not (NC); $R_{2}$ satisfies (NC) and not (WC). In all other respects, $R_{1}$ and $R_{2}$ are identical. This effectively turns the comparison of $R_{1}$ and $R_{2}$ into a comparison between $R_{1}{ }^{*}$ and $R_{2}{ }^{*}$, where both codes only satisfy one constraint: $R_{1}$ * only satisfies (NC) and $R_{2}{ }^{*}$ only satisfies (WC).

Let us compare $R_{1}{ }^{*}$ and $R_{2}{ }^{*}$. Under $R_{1}{ }^{*}, q$ is rationally required of you at all worlds where $p$ holds true, whereas under $R_{2}{ }^{*}(p \rightarrow q)$ is rationally required of you at all worlds. Thus, under both $R_{1}{ }^{*}$ and $R_{2}{ }^{*}$, you violate a rational requirement (and are not fully rational) at $w$ if and only if, at $w, p$ and not- $q$ hold true of you. In all other situations, you are fully rational. Consequently, Broome's Theorem is correct.

But why does this fail to vindicate Property equivalence? Recall that Property equivalence implies that by replacing (NC) with (WC) (or vice versa) in a code $R_{1}$, one creates another code $R_{2}$, which picks out the same set of worlds (and combinations of attitudes) at which you are fully rational as that picked out by $R_{1}$. However, in contrast, Theorem implies that if $R_{1}$ and $R_{2}$ differ only in the described way, then $R_{1}$ and $R_{2}$ pick out an identical set of worlds (or combination of attitudes) at which you are fully rational. The difference is subtle, yet significant.

Here is when it is significant. Suppose you perform only the following operation on a code $R$ : you remove (WC) and inject (NC). As a consequence, this may

\footnotetext{
${ }^{26}$ I cite the (2007a) version of the theorem because it is explicitly about the code of rationality.

${ }^{27}$ This holds true despite an error in the first two versions of his proof (Broome 2007a, pp.369-70; 2007b, pp. 39-40), which Broome successfully corrected in the latest formulation (2013a, p. 148).
} 
entail that the resulting code, call it $R^{*}$, differs in ways that go beyond satisfying (NC) rather than (WC). It may differ in another significant respect. By replacing (WC) with (NC), one may indirectly apply to $R^{*}$ another constraint that does not apply to $R$. This further constraint may manipulate the set of required propositions such that it becomes possible for you to be fully rational under the original but not under the resulting code.

The counterexamples in $\S 4$ and $\S 5$ represent cases in point. Recall my entirely schematic counterexample. In creating $R_{N}$, I followed the instructions of Property equivalence. I removed (WC) from $R_{\mathrm{W}}$ and applied (NC) instead. In doing so, however, I did not create a code that differs from the original code $R_{\mathrm{W}}$ with respect to at most two properties. Instead, I created a code that differs from $R_{\mathrm{W}}$ with respect to more than two properties. By replacing (NC) with (WC), I indirectly injected another constraint - call it (CC) - into $R_{N}$. (CC) reads as follows: 'For all $w:(p \in w) \rightarrow[q \in R P(w)]$ '. But I injected (CC) into $R_{N}$ only indirectly, in virtue of the fact that the constraints on $R_{N}$ are closed under inference by modus ponens. The following table represents this situation:

\section{Code $\left(\mathbf{R}_{\mathrm{w}}\right)$}

(WC) For all $w:(p \rightarrow q) \in R P(w)$.

$(\mathrm{LR})$ For all $w:[q \in R P(w)] \rightarrow[r \in R P(w)]$.

\section{Code $\left(\mathbf{R}_{N}\right)$}

(NC) For all $w:(p \in w) \rightarrow[q \in R P(w)]$.

$(\mathrm{LR})$ For all $w:[q \in R P(w)] \rightarrow[r \in R P(w)]$.

(CC) For all $w:(p \in w) \rightarrow[r \in R P(w)]$.

I showed that under $R_{\mathrm{W}},[p, q$, and not-r] is consistent with being fully rational, while this is not so under $R_{\mathrm{N}}$. Replacing a (WC)-type constraint with an (NC)-type constraint can thus make a difference as to which attitudinal combinations count as fully rational.

However, Theorem evades this result. It does so by stipulating that the two codes can differ only with respect to two particular constraints. Yet $R_{\mathrm{W}}$ and $R_{\mathrm{N}}$ differ with respect to more than two constraints. As a consequence, Theorem disallows the comparison of a (WC)-code and an (NC)-code if these codes satisfy (LR), i.e. one required proposition implying another required proposition. (LR)codes are excluded from Theorem, and this ensures that $R_{\mathrm{W}}$ and $R_{\mathrm{N}}$ thereby fall outside the theorem's scope. Hence, Theorem remains immune to my counterexamples.

But this immunity comes at a heavy cost. As I have argued in $\S 5$, there is no good reason to exclude the existence of (LR)-type constraints on a code. In $\S 5,1$ presented (LR)-type constraints that I find plausible. Also, Broome has not offered any reasons to doubt their existence. This, however, undermines the aim in terms of which Broome advances his theorem. 
Broome advances his theorem in order to demonstrate that '[...] we cannot decide between the wide-scope and narrow-scope formulations by considering when you have the property of [full] rationality' (2007a, p. 364). Under Property equivalence, this would hold true. By excluding (LR)-type requirements, Theorem fails to support it.

Again, the counterexample in $\S 5$ shows this. Here is a schematic version of it. Suppose you believe you ought to $A$ and you do not believe that it is not the case that you ought to $A$. Suppose, further, that you believe that if you do not believe that it is not the case that you ought to $A$, then you do not $X$. Yet you intend to $X$. Are you fully rational?

Suppose the answer is 'yes'. Then, as long as Safety holds, this excludes the correctness of Narrow ought-belief consistency (i.e. if you believe you ought to $A$, then rationality requires of you that you not believe that it is not the case that you ought to A). Narrow ought-belief consistency, in conjunction with Safety, entails that a required proposition fails to hold true of you. Under Narrow ought-belief consistency, you would not be fully rational.

By contrast, suppose that the answer is 'no'. Then, as long as Safety holds, Wide ought-belief consistency (i.e. rationality requires you not to [believe that you ought to $A$ ] and [believe that it is not the case that you ought to $A$ ] simultaneously) cannot be correct, because Wide ought-belief consistency, in conjunction with Safety, does not entail that any required proposition fails to hold true of you. Under Wide ought-belief consistency, you would be fully rational. In sum - pace Broome - the property of full rationality can help to determine whether a conditional requirement takes a wide or a narrow scope. The property of rationality can therefore help us to determine what rationality requires.

\section{Conclusion}

In the debate over the logical form of rational requirements, it has been argued (most prominently by Broome) that the difference between wide- and narrowscope requirements is immaterial to the issue of when a person possesses the property of rationality. Suppose we propose a wide-scope requirement of rationality and replace it with its narrow-scope equivalent. This replacement will not make any difference when it comes to determining when a person is fully rational under the proposed requirement. There is no combination of attitudes that turns out to be rational under a regime of wide-scope requirements that would not turn out to be rational under a regime of equivalent narrow-scope requirements.

In this paper, I show that this is incorrect. First, the equivalence claim cannot be established by Broome's theorem and proof (see $\S 6$ ). Second, replacing a widewith a narrow-scope requirement (or vice versa) can make a difference as to when a person is fully rational. As I have demonstrated in $\S 4$ and $\S 5$, there are 
combinations of attitudes that are rational under a wide-scope requirement but fail to be so under the same narrow-scope requirement. This is the case when the introduction of a narrow-scope requirement triggers the detachment of a further requirement that was not detachable under a wide-scope requirement (as $\S 4$ shows for a schematic code of requirements, and $\S 5$ with an actual code).

This result presents an opportunity for the debate on rational requirements. By following the equivalence claim, we were misled into accepting that we cannot overcome the scope debate by examining whether wide- or narrow-scope requirements give a better account of the property of rationality. But this position is too sceptical. In fact, it deprives us of an important opportunity to make progress in the scope debate. There are situations in which deciding whether you are fully rational will also determine whether a particular requirement has a wide or a narrow logical scope. So, by working out when a person is fully rational, we can also make progress on the question of which logical form represents conditional requirements of rationality.

This does not only mean progress for the scope debate. It will also prove helpful for advancing answers to some of the most important questions linked to the requirements of rationality. Scope and logical form decide, among other things, whether rational requirements can explain the correctness of reasoning, can guide our attitude formation, and are apt to serve as sources of normative reasons.

Perhaps most importantly, the scope of rational requirements decides whether the so-called bootstrapping objection entails a forceful argument against the normativity of rational requirements. As explained in $\S 1$, to assume that narrow-scope requirements are normative leads to incredible bootstrapping. It would licence the spurious fabrication of normative reasons where clearly no such reasons exist. In order to maintain a normative interpretation of rational requirements, these requirements must have a wide logical scope. Thus, until we settle the scope question, the consequences of the bootstrapping objection for the normativity of rational requirements will remain undecided. This underlines why the result of the presented argument is important: if the property of rationality can help us to resolve the scope debate, it will also prove useful for deciding whether or not rational requirements are normative.

Thus, research on rationality should no longer ignore the property of rationality. Indeed, we should try to give an account of the property of rationality that is independent of its requirements - an account on the basis of which we can then determine precisely what rationality requires. ${ }^{28}$

\footnotetext{
${ }^{28}$ Of course, this proposal involves a considerable challenge. We need to establish a way to determine the property of rationality before we formulate the requirements of rationality. That is, we need to find a way to establish the degree of a person's rationality that does not rely on first establishing which and how many rational requirements that person satisfies or violates. For a constructive suggestion on this point, see Fink (2014 and ms).
} 


\section{Appendix}

Kolodny's counterargument. Kolodny (2007b, pp. 375-6) argues that Property equivalence and Theorem do not hold for what he calls 'process requirements' ${ }^{29}$ I argue that Kolodny fails to show this.

Consider a simplified version of a narrow-scope conditional requirement, the wide-scope counterpart of which differs, according to Kolodny, in terms of its conditions of violation: ${ }^{30}$

Necessarily, if, at $t_{1}$, you believe that you ought to $X$, then rationality requires of you that, at $t_{3}$, you intend to $X$,

where time $t_{1}$ precedes $t_{3}$. Expressed as a code constraint, this requirement reads as follows:

(NP) For all $w: B_{t_{1}}[O(X)] \rightarrow\left[I_{t_{3}}(X) \in R P(w)\right]$,

where $B$ stands for 'you believe that', $O$ for 'you ought to', and I for 'you intend to'. The corresponding wide-scope constraint reads as follows:

(WP) For all $w:\left\{B_{t_{1}}[O(X)] \rightarrow I_{t_{3}}(X)\right\} \in R P(w)$.

Kolodny thinks that there are situations in which you are fully rational under (WP), but not so under (NP). His argument runs as follows (2007b, pp. 375-6): suppose that, at $t_{1}$, you believe that you ought to $X$ without intending to $X$. However, at $t_{2}$ (i.e. after $t_{1}$ and before $t_{3}$ ), you abandon your belief that you ought to $X$. Furthermore, at $t_{3}$, you fail to intend to $X$. Kolodny argues that in this situation you cannot be fully rational under (NP). Yet (WP) does not imply this.

This is not correct. It is true that under (NP) you are not entirely rational. Since, at $t_{1}$, you believe that you ought to $X$, 'At $t_{3}$, you intend to $X$ ' is a required proposition. But this proposition turns out to be false. You are not fully rational. However, the same holds for (WP). If, at $t_{1}$, you believe that you ought to $X$, and, at $t_{3}$, you fail to intend to $X$, you are also not entirely as rationality requires you to be, since the required proposition 'If, at $t_{1}$, you believe you ought to $X$, then, at $t_{3}$, you intend to $X^{\prime}$ turns out to be false. This result holds despite the fact

\footnotetext{
${ }^{29}$ Note, first, that Kolodny's 'process requirements' are not exactly process requirements: their contents do not represent a process, nor is a process necessary for their satisfaction (Fink 2011 and 2012). These requirements are in fact diachronic requirements, where rationality requires a crosstemporal relation among attitudes.

${ }^{30}$ I have slightly adapted Kolodny's formulation. Kolodny's original formulation reads as follows: 'Necessarily, if you believe at $t$ that you ought to $X$, but you do not intend at $t$ to $X$, then rationality requires you to form going forward from $t$, on the basis of the content of your belief, the intention to $X^{\prime}$ (2007b, pp. 378-9).
} 
that, at $t_{2}$, you drop your belief that you ought to $X$. Thus Kolodny's example disproves neither Property equivalence nor Theorem.

Broome's proof. Broome (2007a, pp. 369-70) provides us with a proof for his theorem (see § 6). Although Theorem proves correct, Broome's original proof does not. Here is why.

Consider only the final part of the proof. Here, Broome tries to establish that, necessarily, if you are fully rational under $R_{2}$, you are also fully rational under $R_{1}::^{31}$

[T]ake a world $w$ where 'You are rational' is true under $R_{2}$. I shall prove it is also true under $R_{1}$. Since $w$ satisfies all the requirements in $R_{2}(w)$, and $R_{1}(w)$ contains all the same requirements apart from the single one that differs, $w$ satisfies all the requirements in $R_{1}(w)$ apart from, possibly, that final one.

Because $(p \rightarrow q)$ is in $R_{2}(w)$, and 'You are rational' is true at $w$ under $R_{2},(p \rightarrow q)$ is true at $w$. Either $p$ is true at $w$ or it is not. If it is, then $q$ is in $R_{1}(w): q$ is required at $w$ according to $R_{1}$. And this requirement is satisfied; $q$ is true at $w$ because both $p$ and $(p \rightarrow q)$ are true there. On the other hand, if $p$ is not true at $w$, there is no final requirement in $R_{1}(w)$ to be satisfied. Either way, $w$ satisfies all the requirements in $R_{1}(w)$. 'You are rational' is therefore true at $w$ under $R_{1}$.

Broome argues as follows: if $p$ is true at $w$, and you are fully rational under $R_{2}$, then you also satisfy all requirements under $R_{1}$. Under $R_{2},(p \rightarrow q)$ is a required proposition. At all $p$-worlds $(p \rightarrow q)$ is true if and only if $q$ is true. This in turn guarantees that you also satisfy all requirements under $R_{1}$. At all $p$-worlds, $q$ is a required proposition under $R_{1}$.

What about not- $p$-worlds? In those worlds, $(p \rightarrow q)$ is true in virtue of $p$ 's being false. Hence, you are fully rational under $R_{2}$. But what about $R_{1}$ ? Broome argues that you are also fully rational under $R_{1}$. His point is this: 'if $p$ is not true at $w$, there is no final requirement in $R_{1}(w)$ to be satisfied'. That is, if $p$ is false at $w$, then $q$ is not a required proposition.

Consequently, Broome's proof relies on the following principle of requirement 'avoidance'. Described as a constraint of a code, this principle reads as follows:

Avoidance. Necessarily For all $w:\{\{[p \in w] \rightarrow[q \in R P(w)]\}$ $\&[\neg p \in w]\} \rightarrow \neg[q \in R P(w)]$.

Less technically: whenever $p$ implies that rationality requires you to $q$, then if not- $p$, you are not required to $q$.

Avoidance has been commonly assumed to hold true for requirements that are represented by a code satisfying (NC) (see, for example, Broome 2007b, p. 38; 2013a; Lord 2011; Hill 1973; Schroeder 2004, 2005, p. 362; and Vranas 2008).

${ }^{31} R_{1}$ and $R_{2}$ correspond to Broome's Theorem here. 
However, the logic of an (NC)-code fails to support Avoidance. So, to the extent that it relies on Avoidance, Broome's proof contains a mistake.

(NC) depicts the following constraint of a code: necessarily, if $w$ is a $p$-world, then $q$ is a required proposition at $w$. The implication here is a material one. This is necessary, inter alia, to support an important aspect of (WC)-type requirements: if $(p \rightarrow q)$ is a required proposition at $w$, you can satisfy the corresponding requirement by ensuring that 'not- $p$ ' holds at $w$. However, it also implies that the logic of the (NC)-type requirements does not support Avoidance. In fact, Avoidance falls foul of the requirements of logic, as it represents the fallacy of denying the antecedent: the fact that $p$ materially implies that $q$ is a required proposition does not imply that if $p$ is not true, then it is not the case that $q$ is a required proposition.

The following example shows why it would not even be a good idea to inject Avoidance into the logic of requirements that (NC) represents. Suppose you believe that you ought to drive carefully. Suppose this implies materially that 'You to intend to drive carefully' is rationally required of you. At some point you drop your belief that you ought to drive carefully. This does not suffice to ensure that intending to drive carefully is no longer rationally required of you. For example: suppose that you, at the same time, intend to arrive home safe and sound, and you believe that a necessary condition of your doing so is that you drive carefully. Intending to drive carefully will still be rationally required of you - despite your having dropped the belief that you ought to drive carefully. This shows that Avoidance is not correct. Broome's proof contains a mistake. ${ }^{32}$

\footnotetext{
${ }^{32}$ I would like to point out that a similar proof in Broome (2013a, p. 148) does not contain this mistake.
} 


\section{References}

Appiah, Kwame A. 1990, 'Racisms', in D. T. Goldberg, pp. 3-17

Bedke, Matthew 2009, 'The Iffiest Oughts: A Guise of Reasons Account of End - Given Conditionals', in Ethics, 119, pp. 672-98

Bratman, Michael 1987, Intention, Plans, and Practical Reason (Cambridge, MA: Harvard University Press)

Broome, John 1999, 'Normative Requirements', in Ratio, 12, pp. 398-419

- _ 2004, 'Reasons', in Wallace, Jay, Michael Smith, Samuel Scheffler and Philip Pettit, pp. 28-55

- - 2007a, 'Wide or Narrow Scope?', in Mind, 116, pp. 359-70

- - 2007b, 'Requirements', in Toni Rønnow-Rasmussen, Björn Petersson, Jonas Josefsson and Dan Egonsson, pp. 1-41

- - 2007c, 'Is Rationality Normative?', in Disputatio, 2, pp. 161-178

- - 2007d, 'Does Rationality Consist in Responding Correctly to Reasons?', 4, pp. 349-74

_ _ 2013a, Rationality Through Reasoning (Oxford: Wiley-Blackwell)

- 2013b, 'Enkrasia', in Organon F, 20, pp. 425-36

Brunero, John 2010, 'The Scope of Rational Requirements', in Philosophical Quarterly, 60, pp. 28-49

- - 2012, 'Instrumental Rationality, Symmetry and Scope', in Philosophical Studies, 157, pp. 125-40

- - 2013, 'Rational Akrasia', in Organon F, 20, pp. 546-66

Coates, Allen 2013, 'The Enkratic Requirement', in European Journal of Philosophy, 21, pp. 320-333

Evers, Daan 2011, 'Two Objections to Wide-Scoping', in Grazer Philosophische Studien, 83, pp. 251-55

Fink, Julian 2011, 'Are there Process-Requirements of Rationality?', in Organon $F, 18$, pp. 475-87.

Fink, Julian 2012, 'The Function of Normative Process-Requirements', in dialectica, 66, pp. 115-36. 
Fink, Julian 2014, 'A Constitutive Account of "Rationality Requires"', in Erkenntnis, 79, pp. 909-41.

Fink, Julian ms, 'The Essence of Attitudinal Irrationality' (unpublished manuscript).

Goldberg, David T. (ed.) 1990, Anatomy of Racism (Minneapolis: University of Minnesota Press)

Hill, Thomas E. Jr. 1973, 'The Hypothetical Imperative', in The Philosophical Review, 82, pp. 429-50

Kiesewetter, Benjamin 2013, The Normativity of Rationality, Dissertation, Humboldt University of Berlin

Kolodny, Niko 2005, 'Why be Rational?', in Mind, 114, pp. 509-63

- - 2007a, 'How Does Coherence Matter?' in Proceedings of the Aristotelian Society, 107, pp. 229-263

- $-2007 \mathrm{~b}$, 'State or Process Requirements?' in Mind, 116, pp. $371-85$

Lengbeyer, Lawrence. A. 2004, 'Racism and Impure Hearts', in Michael Levine and Tamas Pataki, p. 158-178

Levine, Michael and Tamas Pataki (eds.) 2004, Racism in Mind. Ithaca: Cornell University Press

Lord, Errol 2011, 'Violating Requirements, Exiting from Requirements, and the Scope of Rationality' in Philosophical Quarterly, 243, pp. 392-399

- - 2014a, 'The Coherent and The Rational', in Analytic Philosophy, 55, pp. 151-175

_ - 2014b, 'The Real Symmetry Problem(s) for Wide-Scope Accounts of Rationality' in Philosophical Studies, 170, pp. 443-464

- - forthcoming, 'What You're Rationally Required To Do and What You Ought To Do (Are the Same Thing!)', in Mind

Piller, Christian, 2013, 'The Bootstrapping Objection', in Organon F, 20, pp. 612-31

Reisner, Andrew 2009, in 'Unifying the Requirements of Rationality', in Philosophical Explorations, 12, pp. 243-260 
Rippon, Simon 2011, 'In Defense of the Wide-Scope Instrumental Principle', in Journal of Ethics and Social Philosophy, 5, pp. 1-21

Rønnow-Rasmussen, Toni, Björn Petersson, Jonas Josefsson, and Dan Egonsson (eds.) 2007, Homage à Wlodek: Philosophical Papers Dedicated to Wlodek Rabinowicz, electronic festshrift: www.fil.lu.se/hommageawlodek/

Scanlon, T. M. (2007), 'Structural irrationality', in G. Brennan, R. Goodin, \& M. Smith (Eds.), Common minds (pp. 84-103). Oxford: Oxford University Press.

Schroeder, Mark 2004, 'The Scope of Instrumental Reason', in Philosophical Perspectives, 18, pp. 337-364

- - 2005, 'The Hypothetical Imperative?', in Australasian Journal of Philosophy, 83, pp. 357-372

- - 2009, 'Means-End Coherence, Stringency, and Subjective Reasons' in Philosophical Studies, 143, pp. 223-248

Shpall, Sam 2013, 'Wide and Narrow-Scope', in Philosophical Studies, 163, pp. 717-736

Southwood, Nicholas 2008, in 'Vindicating the Normativity of Rationality', in Ethics, 119, pp. 9-30

Vranas, Peter 2008, 'New Foundations for Imperative Logic I: Logical Connectives, Consistency, and Quantifiers', in Noûs, 42, pp. 529-572

Wallace, Jay, Michael Smith, Samuel Scheffler and Philip Pettit (eds.) 2004: Reason and Value: Themes from the Moral Philosophy of Joseph Raz (Oxford: Oxford University Press)

Way, Jonathan 2010, 'Defending the Wide-Scope Approach to Instrumental Reason', in Philosophical Studies, 147, pp. 213-33

- - 2011, 'The Symmetry of Rational Requirements' in Philosophical Studies, 155, pp. 227-239

Žarnić, Berislav 2010, 'A Logical Typology of Normative Systems', in Journal of Applied Ethics and Philosophy, 2, pp. 30-40 